\title{
1 Utilisation of semiconductor sequencing for detection of actionable fusions in
}

\section{solid tumours}

4 Marco Loddo ${ }^{1 *}$ BSc PhD, Keeda-Marie Hardisty ${ }^{1}$ BSc, Tiffany Haddow ${ }^{1}$ BSc, Robert Thatcher ${ }^{1}$ BSc

5 and Gareth H Williams ${ }^{1 *}$ BSc PhD MBChB FRCPath.

$6{ }^{1}$ Oncologica UK Ltd. Suite 2, The Newnham Building, Chesterford Research Park, Cambridge, CB10 1 XL.

$7 \quad$ * Corresponding authors:

8 Dr Marco Loddo marco.loddo@oncologica.com

9 Prof Gareth Williams gareth.williams@oncologica.com

10 Competing interests: ML and GW are shareholders and directors of Oncologica UK Ltd. ML, KH, TH, RT

11 and GW are currently employed at Oncologica UK Ltd.

12 Abstract:

13 Oncogenic fusions represent compelling druggable targets in solid tumours highlighted by the recent site

14 agnostic FDA approval of larotrectinib for NTRK rearrangements. However screening for fusions in

15 routinely processed tissue samples is constrained due to degradation of nucleic acid as a result of formalin

16 fixation., To investigate the clinical utility of semiconductor sequencing optimised for detection of

17 actionable fusion transcripts in formalin fixed samples, we have undertaken an analysis of test trending

18 data generated by a clinically validated next generation sequencing platform designed to capture 867 of

19 the most clinically relevant druggable driver-partner oncogenic fusions. Here we showacross a real-life

20 cohort of 1112 patients with solid tumours that actionable fusions occur at high frequency (7.4\%) with 
21 linkage to a wide range of targeted therapy protocols including seven fusion-drug matches with FDA/EMA

22 approval and/or NCCN/ESMO recommendations and 80 clinical trials. The majority of actionable fusions identified were independent of tumour type in keeping with signalling via evolutionary conserved Wnt/ $\beta$ catenin, RAS/RAF/MEK/ERK, PI3K/AKT/MTOR, PLCY/PKC and JAK/STAT pathways. Taken together our data indicates that semiconductor sequencing for detection of actionable fusions can be integrated into routine diagnostic pathology workflows enabling the identification of personalised treatment options that

27 have potential to improve clinical cancer management across many tumour types.

\section{Introduction:}

Oncogenic fusion genes are an important class of driver mutation in solid tumors and hematological malignancies [1]. The rationale for targeting gene fusions was first highlighted by the significant clinical responses to imatinib in patients with BCR-ABL-positive chronic myeloid leukemia [2]. This therapeutic approach has now been extended to solid tumours, for example the targeting of ALK, ROS1, NTRK and RET markedly improved survival outcomes [3, 4]. 
44 basket clinical trials in which detection of an actionable genetic variant is used as the determinant for

45 entry into a clinical trial $[8,9]$.

Although many oncogenic fusion genes have now been identified, few are screened as potential

47 therapeutic targets in routine clinical practice. Testing is mostly restricted to the detection of ALK and ROS rearrangements in NSCLC [10]. This relates to the fact that fusion gene analysis is a particular challenge in the clinical context due to the complex combination and numbers of driver genes and partner genes involved in chromosomal rearrangements [1, 11-14]. Moreover the nucleic acid templates extracted from

51 routine formalin fixed paraffin wax embedded tissues (FFPE) biopsy samples are characterised by low DNA/RNA yields with poor integrity and quality which is difficult to sequence particularly in relation to RNA fusion transcripts. Indeed the challenges of genomic profiling of FFPE clinical samples was recently highlighted in the summary report of the Genomic England 100,000 genomes project which utilized fluorescent based sequencing and concluded that analysis of FFPE samples for personalised medicine was infeasible [15].

The increasing use of targeted agents offers the great advantage of increased specificity and reduced toxicity when compared with conventional chemotherapy [16]. Meta-analysis in diverse tumor types has shown that a personalized strategy of treatment is an independent predictor of better outcomes and fewer toxicity associated deaths when compared with chemotherapy [17]. The recent advances in targeted next-generation sequencing (NGS) technologies optimized for nucleic acid templates extracted

62 from FFPE tumour samples now provides the opportunity to conduct precision oncology testing as part 63 of the routine diagnostic work flow. To investigate the potential role of clinically directed semiconductor 64 sequencing in solid tumours we have established a clinically validated NGS platform optimised for analysis of FFPE clinical biopsy samples. This platform enables capture of 764 lead anti-cancer targeted agents/combinations and immunotherapy opportunities via analysis of actionable variants distributed across 505 genes. This screen includes detection of 867 druggable driver-partner oncogenic fusions via 
analysis of 51 driver and 349 partner genes, with linkage to 140 targeted therapy protocols. All variants detected are "actionable" and therefore treatable by targeted therapies either on-market FDA and EMA approved, carrying ESMO and NCCN guideline references or currently in clinical trials, phases I-IV,

71 worldwide [18].

72 Here we have undertaken a retrospective analysis of test trending data to investigate the types and

73 frequency of clinically relevant fusions in solid tumours. Here we show that semiconductor sequencing

74 can be incorporated into routine pathology diagnostic work flows enabling detection of druggable fusions

75 at high frequency across many solid tumour types.

76

\section{Materials and Methods}

Patient demographics:

A retrospective analysis was performed on the trending data generated as part of routine comprehensive

81 requirements for monitoring of quality indicators. The research conducted in this study was limited to

82 secondary use of information previously collected in the course of normal care (without an intention to 83 use it for research at the time of collection) and therefore does not require REC review. The patients and

84 service users were not identifiable to the research team carrying out trend data analysis. This is also in 85 accordance with guidance issued by the National Research Ethics Service, Health Research Authority, NHS

86 and follows the tenants of the Declaration of Helsinki. The trending data relates to a real-life cohort of 871112 patients tested between January 2018 and July 2019. The cancer types and histological classification of the study cohort are shown in S1 Table. 
Sequencing runs were quality controlled using the following parameters according to manufacturer's Comprehensive NGS genomic profiling

The NGS platform utilized for clinical testing is validated for clinical use and accredited by CLIA (ID 99D2170813) and by UKAS (9376) in compliance with ISO15189:2012 and following the guidelines published by the Association for Molecular Pathology and College of American Pathologists and IQN-Path ASBL as described $[8,19]$. The performance characteristics are shown in S2 Table. The NGS platform includes the targeting of 51 driver genes and 349 partner genes enabling detection of 867 druggable driver-partner oncogenic fusions encompassing 400 unique genes and linked to 140 targeted therapy protocols. Genomic regions selected for analysis of clinically relevant fusions are shown in S3 Table.

\section{RNA extraction, library preparation and sequencing:}

RNA was extracted from FFPE curls cut at $10 \mu \mathrm{m}$ or from $5 \mu \mathrm{m}$ sections mounted onto unstained glass slides using the RecoverAll extraction kit (Ambion, Cat no.A26069). RNA samples were diluted to $5 \mathrm{ng} / \mu \mathrm{l}$ and reverse transcribed to cDNA in a 96 well plate using the Superscript Vilo cDNA synthesis kit (CAT 11754250). Library construction, template preparation, template enrichment and sequencing were performed using Ion Ampliseq library 2.0 (Cat:4480441) and the Ion 540TM OT2 kit (Cat: A27753) according to the manufacturer's instructions. Sequencing was performed using the lon S5 system 20 (Cat: A27212) utilising lon 540TM chips (Cat:30 A27766).

\section{Quality control}

110 instructions (Ion Reporter ${ }^{\mathrm{TM}}$ 5.10.1.0): chip loading $>60 \%$ with $>45$ million reads observed, enrichment 98-

$111100 \%$, polyclonal percentage $<55 \%$, low quality $<26 \%$, usable reads $>30 \%$ and aligned bases were $\geq 80 \%$, 
112 unaligned bases were $<20 \%$, mean raw accuracy was $>99 \%$ and overall read length between $100-115 b p$,

113 average base coverage depth $>1200$, uniformity of amplicon (base) coverage $>90 \%$, amplicons were

114 required to have less than $90 \%$ strand bias with $>80 \%$ of amplicons reading end to end, on-target reads

$115>85 \%$ and target base coverage at $1 x, 20 x, 100 x$ and $500 x>90 \%$ (S4 Table).

117 Data analysis:

118 Sequence alignment and variant calling was performed on The Torrent Suite ${ }^{\mathrm{TM}}$ Software (5.8.0). Alignment

119 in Torrent Suite ${ }^{\text {TM }}$ Software was performed using TMAP. The output BAM file was uploaded via the Ion

120 Reporter Uploader plugin (5.8.32-1) to The Ion Reporter ${ }^{\text {TM }}$ Software (5.10.1.0). Gene fusions were

121 reported when occurring in $>40$ counts and meeting the threshold of assay specific internal RNA quality

122 control with a sensitivity of $99 \%$ and PPV of $99 \%$. Six internal expression quality controls were spiked into

123 each sample to monitor assay performance with an acceptance cut-off of $>15$ reads in 5 out 6 controls [lon

124 Reporter ${ }^{\mathrm{TM}}$ 5.10.1.0; default fusion view 5.10] (S3 Table). The results of variant annotation were organized

125 hierarchically by gene, alteration, indication and level of evidence in relation to clinical actionability

126 following the joint recommendation of the association of the AMP/ASCO/CAP [8, 9]. Tertiary analysis

127 software was used to link variants to curated lists of relevant labels, guidelines, and global clinical trials

128 [Oncomine ${ }^{\mathrm{TM}}$ Reporter (Cat:A34298) ]; GlobalData clinical trials database].

\section{Results:}

131 Eighty nine actionable fusion gene events were identified in 1112 samples of solid tumours (Fig 1 and S5

132 Table). Eighty two of the 1112 samples tested had at least one actionable fusion gene representing a

133 frequency of $7.4 \%$. across the study cohort (S1 Table). The frequency of the different gene fusions 
detected is shown in Fig 1. TBL1XR1-PIK3CA, MET-MET, WHSC1L1-FGFR1, EGFR VIII, FGFR3-TACC3 and EGFR-SEPT14 were identified as the most common druggable fusion events (Fig 1). Seven of the samples harboured two fusion genes. Four of these seven cases relate to glioblastoma in which fusion pairs and TBL1XR1-PIK3CA were identified. Fusion pairs were also identified in colon (CAPZA2-MET and METcancers (S4 Table). detected) did show linkage to tumour type (Fig 1 and S5 Table). In tumour types with significant sample size ( $\mathrm{n}>30)$, a high frequency $(>7 \%)$ of actionable fusions was observed in glioblastoma, head and neck

147 cancers, cancers of unknown primary (CUP), prostate and pancreatic cancers and in NSCLC (S6 Table).

148 Glioblastoma, colorectal, lung and breast cancers harboured the most diverse set of actionable fusion genes (Fig 3).

156 (Table 1) either in indication or approved in "cancer of other type" and therefore meeting the tier criteria 157 I and II level of clinical significance as defined by the Joint Consensus Recommendation of the Association 
bioRxiv preprint doi: https://doi.org/10.1101/2021.01.27.428383; this version posted January $27,2021$. The copyright holder for this preprint (which was not certified by peer review) is the author/funder, who has granted bioRxiv a license to display the preprint in perpetuity. It is made available under aCC-BY 4.0 International license.

158 for Molecular Pathology, American Society of Clinical Oncology, and College of American Pathologists [8].

159 These fusions were additionally linked to a total of 80 clinical trials investigating the efficacy of drugs

160 either targeting the kinase fusion directly or alternatively using inhibitors targeting pathways downstream

161 (Table 1).

162 Table 1. Actionable fusion genes in solid tumours.

\begin{tabular}{|c|c|c|c|c|c|}
\hline & & & $\begin{array}{l}\text { Relevant } \\
\text { therapies }\end{array}$ & Clinical trials & Relevant Therapy \\
\hline $\begin{array}{c}\text { Genomic } \\
\text { alteration }\end{array}$ & Cancer type & Pathway & $\begin{array}{c}\text { *approval in } \\
\text { other cancer } \\
\text { type }\end{array}$ & & \\
\hline \multirow[t]{11}{*}{$\begin{array}{l}\text { KANK1 - } \\
\text { NTRK3 }\end{array}$} & Breast & $\begin{array}{l}\text { RAS/RAF/MEK/E } \\
\text { RK }\end{array}$ & entrectinib & NCT02576431 & larotrectinib \\
\hline & & $\begin{array}{l}\text { PI3K/AKT/MTOR } \\
\text { pathway }\end{array}$ & larotrectinib & NCT02637687 & larotrectinib \\
\hline & & PLCy/PKC & & NCT02568267 & entrectinib \\
\hline & & & $\begin{array}{l}\text { *Larotrectinib } \\
\text { (all solid } \\
\text { tumours) }\end{array}$ & NCT02465060 & larotrectinib \\
\hline & & & & NCT03155620 & larotrectinib \\
\hline & & & & NCT02920996 & merestinib \\
\hline & & & & NCT03213704 & larotrectinib \\
\hline & & & & NCT03297606 & temsirolimus \\
\hline & & & & No NCT ID & entrectinib \\
\hline & & & & NCT03093116 & repotrectinib \\
\hline & & & & NCT03215511 & LOXO-195 \\
\hline \multirow[t]{9}{*}{$\begin{array}{c}F G F R 1 \text { - } \\
\text { NRG1 }\end{array}$} & Breast & $\begin{array}{l}\text { RAS/RAF/MEK/E } \\
\text { RK }\end{array}$ & & NCT02052778 & TAS-120 \\
\hline & & $\begin{array}{l}\text { PI3K/AKT/MTOR } \\
\text { pathway }\end{array}$ & & NCT02393248 & $\begin{array}{l}\text { chemotherapy, } \\
\text { INCMGA00012, } \\
\text { pembrolizumab, } \\
\text { pemigatinib, } \\
\text { trastuzumab }\end{array}$ \\
\hline & & PLCy/PKC & & NCT01948297 & FF-284 \\
\hline & & JAK/STAT & & NCT03834220 & FF-284 \\
\hline & & & & NCT02272998 & ponatinib \\
\hline & & & & NCT03297606 & sunitinib \\
\hline & & & & NCT03160833 & HMPL-453 \\
\hline & & & & NCT03929965 & anlotinib hydrochloride \\
\hline & & & & NCT03235570 & pemigatinib \\
\hline \multirow[t]{3}{*}{$\begin{array}{l}\text { TMEM178B } \\
- \text { - MET }\end{array}$} & Colorectal & $\begin{array}{l}\text { RAS/RAF/MEK/E } \\
\text { RK }\end{array}$ & & NCT03297606 & crizotinib \\
\hline & & PI3K/AKT & & NCT03175224 & bozitinib \\
\hline & & & & NCT02219711 & sitravatinib \\
\hline \multirow[t]{2}{*}{$\begin{array}{l}\text { EGFR - } \\
\text { SEPT14 }\end{array}$} & $\begin{array}{l}\text { Colorectal, } \\
\text { Glioblastoma }\end{array}$ & $\begin{array}{l}\text { PI3K/AKT/MTOR } \\
\text { pathway }\end{array}$ & & No NCT ID & $\begin{array}{c}\text { cetuximab + } \\
\text { chemotherapy }\end{array}$ \\
\hline & & $\begin{array}{l}\text { RAS/RAF/MEK/E } \\
\text { RK }\end{array}$ & & No NCT ID & $\begin{array}{c}\text { cetuximab + } \\
\text { chemotherapy }\end{array}$ \\
\hline
\end{tabular}




\begin{tabular}{|c|c|c|c|c|}
\hline & & & NCT03152435 & CART-EGFR \\
\hline & & & NCT03454620 & $\begin{array}{c}\text { GC1118A + } \\
\text { chemotherapy }\end{array}$ \\
\hline & & & NCT03319459 & $\begin{array}{c}\text { cetuximab + FATE- } \\
\text { NK100 }\end{array}$ \\
\hline & & & NCT03297606 & erlotinib \\
\hline & & & NCT02013089 & erlotinib, gefitinib \\
\hline & & & NCT02423525 & afatinib \\
\hline \multirow[t]{4}{*}{$\begin{array}{l}\text { CAPZA2 - } \\
\quad \text { MET }\end{array}$} & $\begin{array}{c}\text { Glioblastoma, } \\
\text { Colorectal }\end{array}$ & $\begin{array}{l}\text { RAS/RAF/MEK/E } \\
\text { RK }\end{array}$ & NCT03598244 & volitinib \\
\hline & & PI3K/AKT & NCT03297606 & crizotinib \\
\hline & & & NCT03175224 & bozitinib \\
\hline & & & NCT02219711 & sitravatinib \\
\hline \multirow[t]{2}{*}{$\begin{array}{l}\text { FIP1L1 - } \\
\text { PDGFRA }\end{array}$} & Glioblastoma & $\begin{array}{l}\text { RAS/RAF/MEK/E } \\
\text { RK }\end{array}$ & NCT03352427 & dasatinib, everolimus \\
\hline & & $\begin{array}{l}\text { PI3K/AKT/MTOR } \\
\text { pathway }\end{array}$ & & \\
\hline \multirow[t]{4}{*}{$\begin{array}{l}\text { PTPRZ1 - } \\
\quad \text { MET }\end{array}$} & Glioblastoma & $\begin{array}{l}\text { RAS/RAF/MEK/E } \\
\text { RK }\end{array}$ & NCT03598244 & volitinib \\
\hline & & PI3K/AKT & NCT03297606 & crizotinib \\
\hline & & & NCT03175224 & bozitinib \\
\hline & & & NCT02219711 & sitravatinib \\
\hline \multirow[t]{9}{*}{$A G K-B R A F$} & Glioblastoma & $\begin{array}{l}\text { RAS/RAF/MEK/E } \\
\text { RK }\end{array}$ & NCT02639546 & cobimetinib \\
\hline & & & NCT02029001 & sorafenib \\
\hline & & & NCT03843775 & $\begin{array}{l}\text { binimetinib + } \\
\text { encorafenib }\end{array}$ \\
\hline & & & NCT03520075 & ASTX029 \\
\hline & & & NCT03905148 & lifirafenib, PD-0325901 \\
\hline & & & NCT03415126 & ASN007 \\
\hline & & & NCT02857270 & $\begin{array}{l}\text { abemaciclib, cetuximab, } \\
\text { chemotherapy, } \\
\text { encorafenib, } \\
\text { LY3214996, midazolam }\end{array}$ \\
\hline & & & NCT02607813 & LXH 254 \\
\hline & & & NCT03634982 & RMC-4630 \\
\hline \multirow[t]{3}{*}{$\begin{array}{l}\text { TBL1XR1 - } \\
\text { PIK3CA }\end{array}$} & $\begin{array}{c}\text { Breast, } \\
\text { Cervical, } \\
\text { Colorectal, } \\
\text { CUP, } \\
\text { endometrial, } \\
\text { gastric, } \\
\text { glioblastoma, } \\
\text { head and } \\
\text { neck, GOJ, } \\
\text { NSCLC, } \\
\text { Oesophageal, } \\
\text { Ovarian, } \\
\text { Pancreatic, } \\
\text { Thyroid, } \\
\text { Vulva }\end{array}$ & $\begin{array}{l}\mathrm{PI} 3 \mathrm{~K} / \mathrm{AKT} / \mathrm{MTOR} \\
\text { pathway }\end{array}$ & NCT03292250 & alpelisib \\
\hline & & & NCT03065062 & gedatolisib + palbociclib \\
\hline & & & NCT03297606 & temsirolimus \\
\hline
\end{tabular}




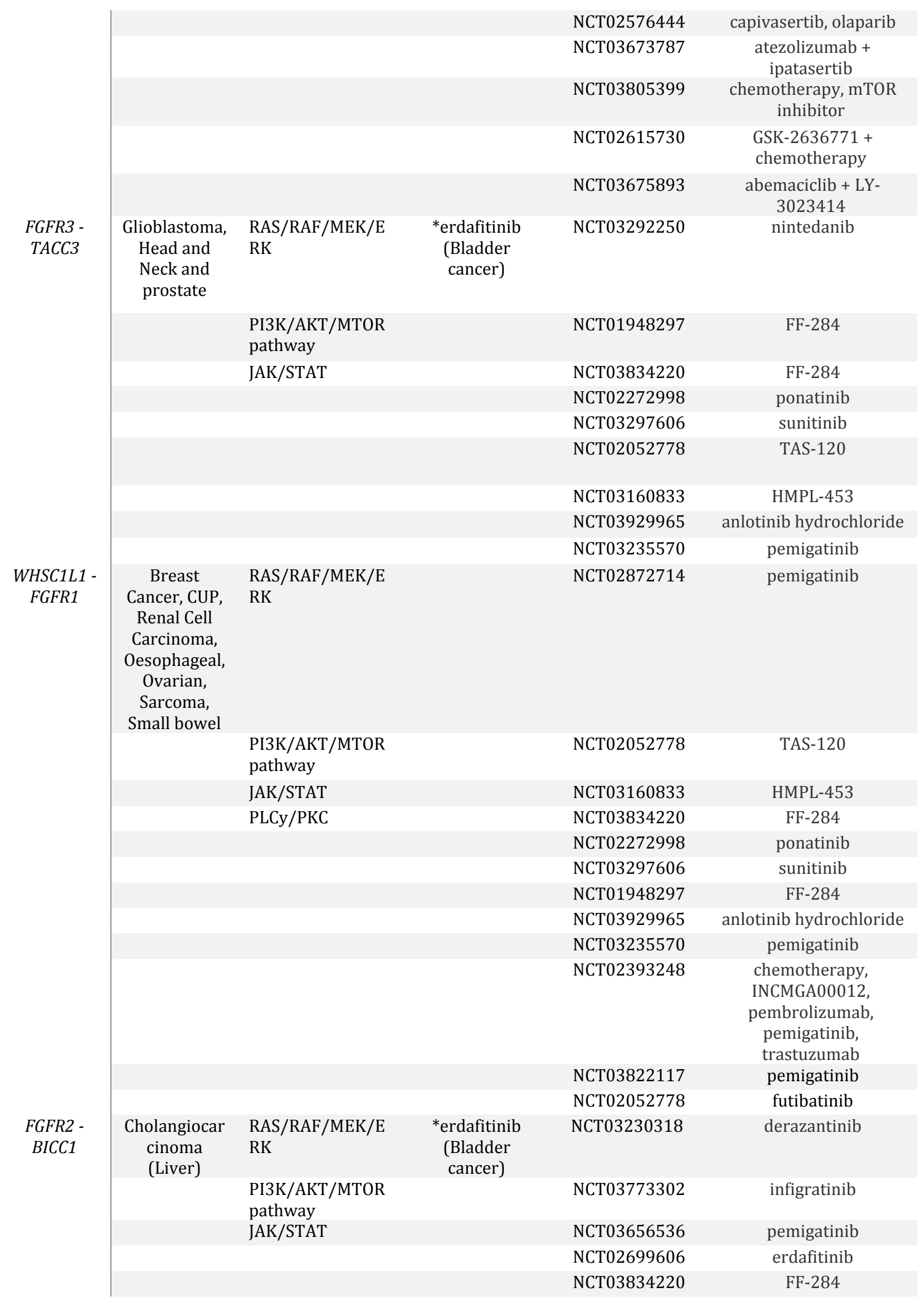




\begin{tabular}{|c|c|c|c|c|c|}
\hline & & & & NCT02150967 & infigratinib \\
\hline & & & & NCT02924376 & pemigatinib \\
\hline & & & & NCT02691793 & sunitinib \\
\hline & & & & NCT02272998 & ponatinib \\
\hline & & & & NCT03297606 & sunitinib \\
\hline & & & & NCT02052778 & TAS-120 \\
\hline & & & & NCT02393248 & $\begin{array}{l}\text { chemotherapy, } \\
\text { INCMGA00012, } \\
\text { pembrolizumab, } \\
\text { pemigatinib, } \\
\text { trastuzumab }\end{array}$ \\
\hline & & & & NCT03160833 & HMPL-453 \\
\hline & & & & NCT01948297 & FF-284 \\
\hline & & & & NCT03929965 & anlotinib hydrochloride \\
\hline & & & & NCT03235570 & pemigatinib \\
\hline KIF5B - RET & NSCLC & $\begin{array}{l}\mathrm{PI} 3 \mathrm{~K} / \mathrm{AKT} / \mathrm{MT} \\
\text { OR }\end{array}$ & Cabozantinib & No NCT ID & alectinib \\
\hline & & $\begin{array}{l}\text { RAS/RAF/MEK/E } \\
\text { RK }\end{array}$ & vandetanib & NCT03194893 & alectinib, crizotinib \\
\hline & & PLCy/PKC & & NCT03391869 & $\begin{array}{l}\text { ipilimumab, nivolumab, } \\
\text { radiation therapy, } \\
\text { surgical intervention }\end{array}$ \\
\hline & & & & NCT01639508 & cabozantinib \\
\hline & & & & NCT02314481 & alectinib \\
\hline & & & & NCT03445000 & alectinib \\
\hline & & & & NCT02540824 & apatinib \\
\hline & & & & NCT02699606 & erdafitinib \\
\hline & & & & NCT02299141 & nintedanib \\
\hline & & & & NCT02664935 & sitravatinib \\
\hline & & & & No NCT ID & $\begin{array}{l}\text { targeted therapy, } \\
\text { targeted therapy + } \\
\text { chemotherapy }\end{array}$ \\
\hline & & & & NCT03157128 & LOXO-292 \\
\hline & & & & NCT03037385 & BLU-667 \\
\hline & & & & NCT03780517 & BOS172738 \\
\hline & & & & NCT02219711 & sitravatinib \\
\hline & & & & NCT02029001 & sorafenib \\
\hline & & & & NCT02450123 & sunitinib \\
\hline & & & & NCT02691793 & sunitinib \\
\hline & & & & NCT02272998 & ponatinib \\
\hline & & & & NCT03297606 & sunitinib \\
\hline SND1 - MET & NSCLC & $\begin{array}{l}\text { RAS/RAF/MEK/E } \\
\text { RK }\end{array}$ & & NCT03088930 & crizotinib \\
\hline & & PI3K/AKT & & NCT02323126 & capmatinib + nivolumab \\
\hline & & & & NCT02414139 & capmatinib \\
\hline & & & & NCT02219711 & sitravatinib \\
\hline & & & & NCT03297606 & crizotinib \\
\hline & & & & NCT03175224 & bozitinib \\
\hline $\begin{array}{c}\text { RET - } \\
\text { NCOA4 }\end{array}$ & NSCLC & $\begin{array}{l}\mathrm{PI} 3 \mathrm{~K} / \mathrm{AKT} / \mathrm{MT} \\
\text { OR }\end{array}$ & cabozantinib & No NCT ID & alectinib \\
\hline
\end{tabular}




\begin{tabular}{|c|c|c|c|c|c|}
\hline & & $\begin{array}{l}\text { RAS/RAF/MEK/E } \\
\text { RK }\end{array}$ & vandetanib & NCT03194893 & alectinib, crizotinib \\
\hline & & PLCy/PKC & & NCT03391869 & $\begin{array}{l}\text { ipilimumab, nivolumab, } \\
\text { radiation therapy, } \\
\text { surgical intervention }\end{array}$ \\
\hline & & & & NCT02314481 & alectinib \\
\hline & & & & NCT03445000 & alectinib \\
\hline & & & & NCT02540824 & apatinib \\
\hline & & & & NCT01639508 & cabozantinib \\
\hline & & & & NCT02699606 & erdafitinib \\
\hline & & & & NCT02299141 & nintedanib \\
\hline & & & & NCT02664935 & sitravatinib \\
\hline & & & & No NCT ID & $\begin{array}{l}\text { targeted therapy, } \\
\text { targeted therapy }+ \\
\text { chemotherapy }\end{array}$ \\
\hline & & & & NCT03157128 & LOXO-292 \\
\hline & & & & NCT03037385 & BLU-667 \\
\hline & & & & NCT03780517 & BOS172738 \\
\hline & & & & NCT02219711 & sitravatinib \\
\hline & & & & NCT02029001 & sorafenib \\
\hline & & & & NCT02450123 & sunitinib \\
\hline & & & & NCT02691793 & sunitinib \\
\hline & & & & NCT02272998 & ponatinib \\
\hline & & & & NCT03297606 & sunitinib \\
\hline $\begin{array}{l}\text { CCDC6 - } \\
\text { RET }\end{array}$ & $\begin{array}{l}\text { NSCLC, } \\
\text { Thyroid }\end{array}$ & $\begin{array}{l}\mathrm{PI} 3 \mathrm{~K} / \mathrm{AKT} / \mathrm{M} \\
\text { TOR }\end{array}$ & $\begin{array}{l}\text { cabozantinib } \\
\text { (NSCLC) }\end{array}$ & No NCT ID & alectinib \\
\hline & & $\begin{array}{l}\text { RAS/RAF/MEK/E } \\
\text { RK }\end{array}$ & $\begin{array}{l}\text { vandetanib } \\
\text { (NSCLC) }\end{array}$ & NCT03194893 & alectinib, crizotinib \\
\hline & & PLCy/PKC & & NCT03391869 & $\begin{array}{l}\text { ipilimumab, nivolumab, } \\
\text { radiation therapy, } \\
\text { surgical intervention }\end{array}$ \\
\hline & & & & NCT02314481 & alectinib \\
\hline & & & & NCT03445000 & alectinib \\
\hline & & & & NCT02540824 & apatinib \\
\hline & & & & NCT01639508 & cabozantinib \\
\hline & & & & NCT02699606 & erdafitinib \\
\hline & & & & NCT02299141 & nintedanib \\
\hline & & & & NCT02664935 & sitravatinib \\
\hline & & & & No NCT ID & $\begin{array}{l}\text { targeted therapy, } \\
\text { targeted therapy + } \\
\text { chemotherapy }\end{array}$ \\
\hline & & & & NCT03157128 & LOXO-292 \\
\hline & & & & NCT03037385 & BLU-667 \\
\hline & & & & NCT03780517 & BOS172738 \\
\hline & & & & NCT02219711 & sitravatinib \\
\hline & & & & NCT02029001 & sorafenib \\
\hline & & & & NCT02450123 & sunitinib \\
\hline & & & & NCT02691793 & sunitinib \\
\hline & & & & NCT02272998 & ponatinib \\
\hline
\end{tabular}




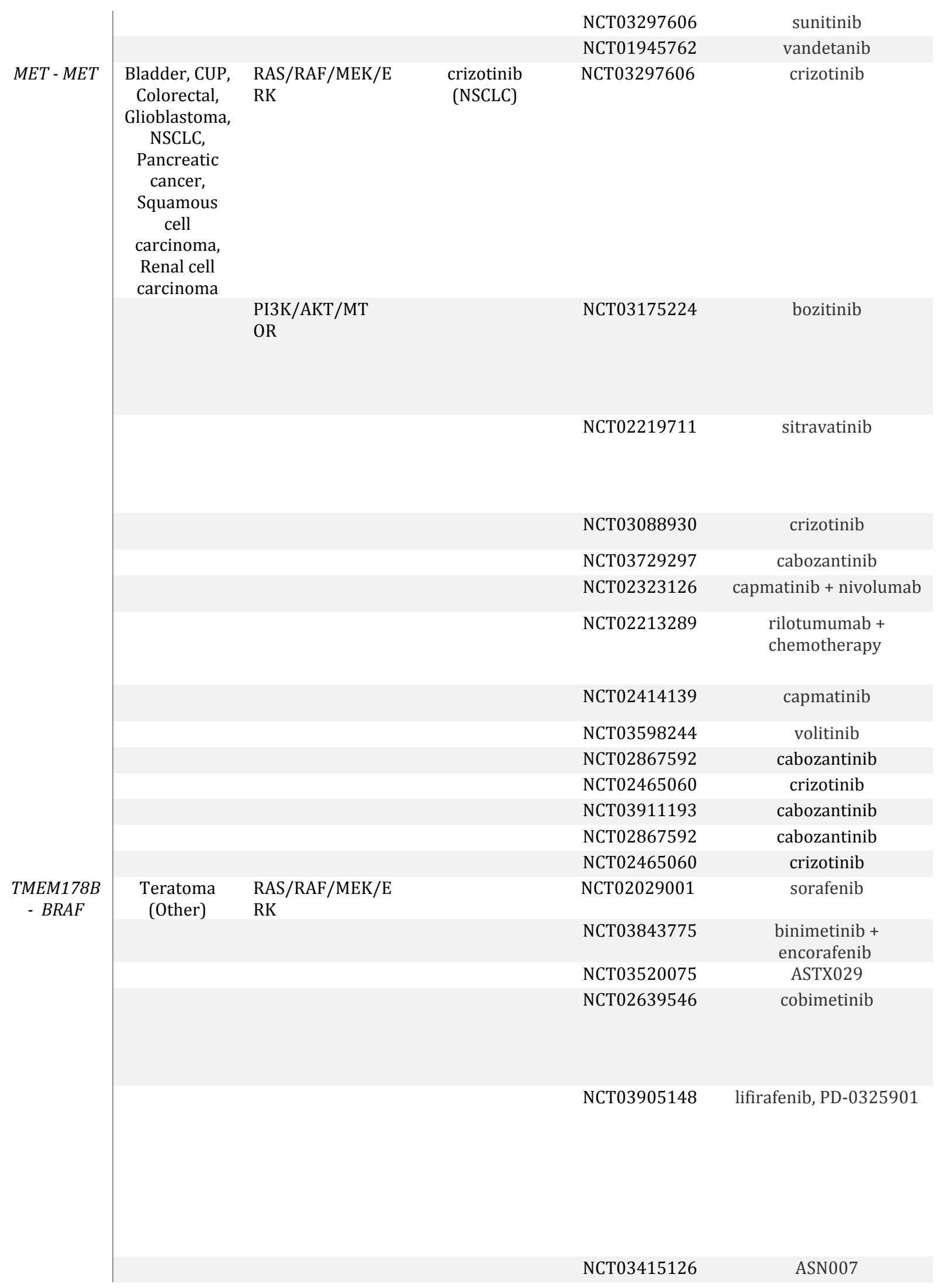




\begin{tabular}{|c|c|c|c|c|}
\hline & & & NCT02857270 & $\begin{array}{c}\text { abemaciclib, cetuximab, } \\
\text { chemotherapy, } \\
\text { encorafenib, } \\
\text { LY3214996, midazolam }\end{array}$ \\
\hline & & & NCT02607813 & LXH254 \\
\hline & & & NCT03634982 & RMC-4630 \\
\hline & & & NCT02013089 & sorafenib, sunitinib \\
\hline & & & NCT03714958 & HDM-201 + trametinib \\
\hline $\begin{array}{c}F N D C 3 B- \\
P I K 3 C A\end{array}$ & Pancreas & $\begin{array}{l}\text { PI3K/AKT/MTOR } \\
\text { pathway }\end{array}$ & NCT03065062 & gedatolisib + palbociclib \\
\hline & & & NCT03297606 & temsirolimus \\
\hline & & & NCT02576444 & capivasertib, olaparib \\
\hline & & & NCT03673787 & $\begin{array}{l}\text { atezolizumab + } \\
\text { ipatasertib }\end{array}$ \\
\hline $\begin{array}{l}\text { SND1 - } \\
\text { BRAF }\end{array}$ & Prostate & $\begin{array}{l}\text { RAS/RAF/MEK/E } \\
\text { RK }\end{array}$ & NCT02029001 & sorafenib \\
\hline & & & NCT03843775 & $\begin{array}{l}\text { binimetinib + } \\
\text { encorafenib }\end{array}$ \\
\hline & & & NCT03520075 & ASTX029 \\
\hline & & & NCT02639546 & cobimetinib \\
\hline & & & NCT03905148 & lifirafenib, PD-0325901 \\
\hline & & & NCT03415126 & ASN007 \\
\hline & & & NCT02857270 & $\begin{array}{l}\text { abemaciclib, cetuximab, } \\
\text { chemotherapy, } \\
\text { encorafenib, } \\
\text { LY3214996, midazolam }\end{array}$ \\
\hline & & & NCT02607813 & LXH254 \\
\hline & & & NCT03634982 & RMC-4630 \\
\hline $\begin{array}{l}\text { BRAF - } \\
\text { MRPS33 }\end{array}$ & Prostate & $\begin{array}{l}\text { RAS/RAF/MEK/E } \\
\text { RK }\end{array}$ & NCT02029001 & sorafenib \\
\hline & & & NCT03843775 & $\begin{array}{l}\text { binimetinib + } \\
\text { encorafenib }\end{array}$ \\
\hline & & & NCT03520075 & ASTX029 \\
\hline & & & NCT02639546 & cobimetinib \\
\hline & & & NCT03905148 & lifirafenib, PD-0325901 \\
\hline & & & NCT03415126 & ASN007 \\
\hline & & & NCT02857270 & $\begin{array}{c}\text { abemaciclib, cetuximab, } \\
\text { chemotherapy, } \\
\text { encorafenib, } \\
\text { LY3214996, midazolam }\end{array}$ \\
\hline & & & NCT02607813 & LXH254 \\
\hline & & & NCT03634982 & RMC-4630 \\
\hline $\begin{array}{l}E G F R- \\
E G F R\end{array}$ & $\begin{array}{l}\text { Glioblastoma, } \\
\text { Prostate }\end{array}$ & $\begin{array}{l}\text { PI3K/AKT/MTOR } \\
\text { pathway }\end{array}$ & NCT03297606 & erlotinib \\
\hline & & $\begin{array}{l}\text { RAS/RAF/MEK/E } \\
\text { RK }\end{array}$ & NCT02423525 & afatinib \\
\hline & & & NCT03319459 & $\begin{array}{c}\text { cetuximab + FATE- } \\
\text { NK100 }\end{array}$ \\
\hline
\end{tabular}




\section{3}

\begin{tabular}{|c|c|c|c|c|}
\hline \multirow[t]{9}{*}{$\begin{array}{l}\text { PCM1 - } \\
B R A F\end{array}$} & Sarcoma & $\begin{array}{l}\text { RAS/RAF/MEK/E } \\
\text { RK }\end{array}$ & NCT02639546 & cobimetinib \\
\hline & & & NCT02029001 & sorafenib \\
\hline & & & NCT03843775 & $\begin{array}{l}\text { binimetinib + } \\
\text { encorafenib }\end{array}$ \\
\hline & & & NCT03520075 & ASTX029 \\
\hline & & & NCT03905148 & lifirafenib, PD-0325901 \\
\hline & & & NCT03415126 & ASN007 \\
\hline & & & NCT02857270 & $\begin{array}{l}\text { abemaciclib, cetuximab, } \\
\text { chemotherapy, } \\
\text { encorafenib, } \\
\text { LY3214996, midazolam }\end{array}$ \\
\hline & & & NCT02607813 & LXH254 \\
\hline & & & NCT03634982 & RMC-4630 \\
\hline $\begin{array}{l}\text { PTPRK - } \\
\text { RSPO3 }\end{array}$ & Colorectal & $\begin{array}{l}\text { WNT/ } \beta \\
\text { catenin }\end{array}$ & NCT01351103 & LGK974 \\
\hline $\begin{array}{l}\text { EIF3E - } \\
\text { RSPO2 }\end{array}$ & Colorectal & $\begin{array}{l}\text { WNT/ } \beta \\
\text { catenin }\end{array}$ & NCT01351103 & LGK974 \\
\hline
\end{tabular}

164 Fig 1. Actionable fusion gene landscape in solid tumours.

165 Fig 2. Frequency of the four most common actionable gene fusions in solid tumours.

166 Fig 3. Frequency of actionable gene fusions in glioblastoma, colorectal, NSCLC and breast cancers.

\section{Discussion:}

168 Advances in somatic cancer genetics and genomic profiling is leading to a shift in treatment paradigm

169 from relatively non-specific empirically directed cytotoxic chemotherapies to a more biologically informed

170 targeted approach [20-22]. The drug-target pairing that links a dysregulated molecular pathway with a

171 cognate therapeutic agent defines the modern era of precision oncology. Targeted agents and

172 immunotherapies are associated with superior response rates, fewer side effects and reduced healthcare

173 costs when compared with nonselective chemotherapy [16, 17, 23].

174 Analysis of our NGS test trending data has shown that actionable fusions occur across a wide range of

175 tumour types providing more personalised treatment options to cancer patients. Actionable fusions were

176 identified at high frequency, $7.3 \%$ across all solid tumour types, rising to $23 \%$ in the case of glioblastoma.

177 Notably the majority of high frequency fusions were agnostic of tumour type. A proportion of low 
frequency fusions did however exhibit tumour type specificity including CCDC6-RET fusions in thyroid and lung cancer, FGFR2-BICC1 in cholangiocarcinoma, PTPRZ1-MET in glioblastoma, EIF3E-RSPO2 and PTPRKRSPO3 in colorectal cancer and KIF5B-RET in NSCLC [24-28]. Other low frequency fusions namely, AGKBRAF, FIP1L1-PDGFRA, FNDC3B-PIK3CA, RET-NCOA4, SND1-BRAF, TMEM178B-MET, TMEM178B-BRAF, KANK1-NTRK3, EIF3E-RSPO2 and PTPRK -RSPO3 have been previously reported as rare fusions in tumours of other type [27, 29-35]. Non targeted novel fusions were also identified including FGFR1-NRG1 in breast, BRAF-MRPS33 in prostate, SND1-MET in lung, PCM1-BRAF in sarcoma and TMEM178B-MET in rectal cancer.

Detected actionable fusions were found to interact with one or more of the major evolutionary conserved cell signalling pathways, namely RAS/RAF/MEK/ERK, PI3K/AKT/MTOR, PLCy/PKC, JAK/STAT and WNT/ $\beta$ catenin. These are key signalling networks mediating fundamental processes including cell proliferation, differentiation, cell migration and apoptosis which are involved in homeostasis across all tissue types [36]. This may account for the fact that the majority of detected actionable fusions are independent of tumour type or tissue of origin. The minority population of tumour specific fusions identified also involve interaction with these conserved signalling networks suggesting that the tumour specificity of these particular fusions does not relate to tissue specific signalling pathways. Moreover tissue specificity does not appear to be determined by tissue specific expression of the partner or driver genes involved in these fusions. For example, in the case of KIF5B-RET in NSCLC, KIF5B is expressed across a broad range of tissue types in keeping with its ubiquitous function as key component of the mitotic machinery and similarly RET is expressed across a broad range of tissue types $[37,38]$. The mechanisms relating to tissue specificity remain poorly defined but may relate to other factors, for example interphase gene proximity (spatial proximity) that can facilitate generation of fusion genes $[11,39,40]$. Interestingly some fusions were associated with a particular tumour subtype, for example all fusions detected in breast cancer were 
202

restricted to cancers of ductal type and in lung cancers to those of NSCLC type. Cellular morphology has been postulated to represent a holistic readout of the complex genomic and gene expression changes in cancer cells and it is interesting to speculate that activation of the cognate signalling pathways linked to these particular fusions drive genomic changes and transcriptome profiles that act as determinants of these specific morpho-phenotypes [41].

The majority of detected druggable oncogenic driver genes represent tyrosine kinases (TKs) with partner genes encoding coil coiled domains leading to ligand-independent homodimerization, TK activation and dysregulated growth. However, it is also possible that disruption of the partner gene itself may also contribute to tumourigenesis through putative tumour suppressor roles. A number of the partner genes identified including TACC, CCDC6, EIF3E and KIF5B participate in DNA damage repair or mitotic chromosomal segregation [42-44]. Dysregulation of these genes have potential to drive error-prone DNA replication leading to genomic instability. This is in keeping with the notion that gene fusion events may function as a "two hit" model for multistep tumourigensis.

Currently routine clinical molecular testing in relation to druggable fusion genes is limited to NSCLC, but even in this tumour type, analysis covers only a small number of potential driver and partner genes, namely ALK and ROS rearrangements [10]. Here we have shown that semiconductor sequencing analysis of fusion transcripts in routine FFPE samples applied across all solid tumour types enables comprehensive analysis of hundreds of druggable fusion genes. Their detection enables clinical evidence based linkage to a broad therapeutic armamentarium of targeted therapies which can have a major impact in the improved clinical management of advanced solid tumours. The majority of targeted agents are directed against the fusions themselves but alternatively treatment protocols also include targeting components of the cognate signalling pathways downstream. For example, the TBL1XR1-PIK3CA fusion is linked to targeted 
therapies inhibiting PI3K directly (alpelisib) but also inhibitors targeting downstream signalling

226 components including AKT (capivasertib), MTOR temsirolimus) and CDK4/6 (abemaciclib). Although a

227 targeted agent for TRK fusions now has FDA approval, this rearrangement was identified as a rare event

228 in our cohort of solid tumours (<1\%) in keeping wFith previous reports [1]. In contrast here we have shown

229 that targeting high frequency fusions such as TBL1XR1-PIK3CA, MET-MET, WHSC1L1-FGFR1 fusions offer

230 much broader clinical utility across all tumour types.

231 In summary we have shown that NGS semiconductor sequencing optimised for analysis druggable fusions

232 occur at high frequency in solid tumours and that their coverage is therefore an important component of

233 comprehensive precision oncology profiling, thereby ensuring that all targeted therapeutic opportunities

234 are captured. Notably, the majority of actionable fusions are not tumor type specific reinforcing the "site

235 agnostic" approach to genomic profiling and supporting the concept of "molecular basket" clinical trials.

236 Importantly we have also demonstrated that adoption of semiconductor sequencing methodologies

237 enables comprehensive precision oncology profiling to be applied robustly to routine FFPE clinical biopsy

238 samples allowing integration with globally established routine diagnostic pathology workflows.

\section{Author contributions}

$\mathrm{ML}$ and GW were responsible for study design, conceptualization, supervision and manuscript preparation. $\mathrm{RT}$, $\mathrm{TH}$ and $\mathrm{KH}$ performed the data analysis and visualization.

\section{Data availability}

247 details are available from the corresponding author upon reasonable request. 


\section{Ethical statement}

250 The research conducted in this study was limited to secondary use of information previously collected in

251 the course of normal care (without an intention to use it for research at the time of collection) and

252 therefore does not require REC review. The patients and service users were not identifiable to the

253 research team carrying out trend data analysis. This is also in accordance with guidance issued by the

254 National Research Ethics Service, Health Research Authority, NHS and follows the tenants of the

255 Declaration of Helsinki.

256

257 Acknowledgements

258

259 The authors thank the technical support staff at Oncologica UK Ltd for their valuable contribution to the

260 study.

261

262

263

264

265

266

267

268

269 


\section{REFERENCES}

271 1. Stransky N, Cerami E, Schalm S, Kim JL, Lengauer C. The landscape of kinase fusions in cancer. Nat

$272 \quad$ Commun. 2014;5: 4846.

273

2. Druker BJ, Talpaz M, Resta DJ, Peng B, Buchdunger E, Ford JM, et al. Efficacy and safety of a specific inhibitor of the BCR-ABL tyrosine kinase in chronic myeloid leukemia. N Engl J Med. 2001;344(14):

275 1031-1037.

3. Ferrara R, Auger N, Auclin E, Besse B. Clinical and Translational Implications of RET Rearrangements in Non-Small Cell Lung Cancer. J Thorac Oncol. 2018;13(1): 27-45.

4. Cheng TY, Cramb SM, Baade PD, Youlden DR, Nwogu C, Reid ME. The International Epidemiology of Lung Cancer: Latest Trends, Disparities, and Tumor Characteristics. J Thorac Oncol. 2016;11(10): 1653-1671.

5. Jørgensen JT. A paradigm shift in biomarker guided oncology drug development. Ann Transl Med. 2019;7(7): 148.

6. Scott LJ. Larotrectinib: First Global Approval. Drugs. 2019;79(2): 201-206.

7. Lemery S, Keegan P, Pazdur R. First FDA Approval Agnostic of Cancer Site - When a Biomarker Defines the Indication. N Engl J Med. 2017;377(15): 1409-1412.

8. Li MM, Datto M, Duncavage EJ, Kulkarni S, Lindeman NI, Roy S, et al. Standards and Guidelines for the Interpretation and Reporting of Sequence Variants in Cancer: A Joint Consensus Recommendation of American Pathologists. J Mol Diagn. 2017;19(1): 4-23. 
9. Mateo J, Chakravarty D, Dienstmann R, Jezdic S, Gonzalez-Perez A, Lopez-Bigas N, et al. A framework to rank genomic alterations as targets for cancer precision medicine: the ESMO Scale for Clinical Actionability of molecular Targets (ESCAT). Ann Oncol. 2018;29(9): 1895-1902.

10. Garinet S, Laurent-Puig P, Blons H, Oudart JB. Current and Future Molecular Testing in NSCLC, What Can We Expect from New Sequencing Technologies?. J Clin Med. 2018;7(6): 144.

11. Tuna $\mathrm{M}, \mathrm{Amos} \mathrm{Cl}$, Mills $\mathrm{GB}$. Molecular mechanisms and pathobiology of oncogenic fusion transcripts in epithelial tumors. Oncotarget. 2019;10(21): 2095-2111.

12. Gao Q, Liang WW, Foltz SM, Mutharasu G, Jayasinghe RG, Cao S, et al. Driver Fusions and Their Implications in the Development and Treatment of Human Cancers. Cell Rep. 2018;23(1): 227-238.e3.

13. Barr FG. Fusion genes in solid tumors: the possibilities and the pitfalls. Expert Rev Mol Diagn. 2016;16(9): 921-923.

14. Latysheva NS, Babu MM. Discovering and understanding oncogenic gene fusions through data intensive computational approaches. Nucleic Acids Res. 2016;44(10): 4487-4503.

15. Turnbull C. Introducing whole-genome sequencing into routine cancer care: the Genomics England 100000 Genomes Project. Ann Oncol. 2018;29(4): 784-787.

16. Haslem DS, Chakravarty I, Fulde G, Gilbert H, Tudor BP, Lin K, et al. Precision oncology in advanced cancer patients improves overall survival with lower weekly healthcare costs. Oncotarget. 2018;2;9(15): 12316-12322.

17. Schwaederle M, Zhao M, Lee JJ, Eggermont AM, Schilsky RL, Mendelsohn J, et al. Impact of Precision Medicine in Diverse Cancers: A Meta-Analysis of Phase II Clinical Trials. J Clin Oncol. 2015; 10;33(32): 3817-25. 
18. Loddo M, Hardisty KM, Thatcher RP, Haddow TE, Williams GH. The actionable genomic mutational landscape in solid tumours. J Clin Oncol. 2020;38(15): suppl.e13642.

19. Deans ZC, Costa JL, Cree I, Dequeker E, Edsjö A, Henderson S, et al. IQN Path ASBL. Integration of nextgeneration sequencing in clinical diagnostic molecular pathology laboratories for analysis of solid

20. Gaines ME. Charting a Course: Navigating the Hype and the Hope of Precision Medicine Together. ASCO Guest commentary. Available at https://connection.asco.org/blogs/charting-course-navigatinghype-and-hope-precision-medicine-together. Deposited 5 August, 2019.

21. Marquart J, Chen EY, Prasad V. Estimation of the Percentage of US Patients With Cancer Who Benefit From Genome-Driven Oncology. JAMA Oncol. 2018;4(8): 1093-1098.

22. Tsimberidou AM, Hong DS, Ye Y, Cartwright C, Wheler JJ, Falchook GS, et al. Initiative for Molecular Profiling and Advanced Cancer Therapy (IMPACT): An MD Anderson Precision Medicine Study. JCO Precis Oncol. 2017;2017.

23. Gong J, Pan K, Fakih M, Pal S, Salgia R. Value-based genomics. Oncotarget. 2018;9(21): 15792-15815.

24. Okamoto K, Kodama K, Takase K, Sugi NH, Yamamoto Y, Iwata M, et al. Antitumor activities of the targeted multi-tyrosine kinase inhibitor lenvatinib (E7080) against RET gene fusion-driven tumor

25. Ying X, Tu J, Wang W, Li X, Xu C, Ji J. FGFR2-BICC1: A Subtype Of FGFR2 Oncogenic Fusion Variant In Cholangiocarcinoma And The Response To Sorafenib. Onco Targets Ther. 2019;12: 9303-9307.

26. Bao ZS, Chen HM, Yang MY, Zhang C-B, Yu K, Ye W-L, et al. RNA-seq of 272 gliomas revealed a novel, recurrent PTPRZ1-MET fusion transcript in secondary glioblastomas. Genome Res. 2014;24(11): 1765-1773. 
27. Seshagiri S, Stawiski EW, Durinck S, Modrusan Z, Storm EE, Conboy CB, et al. Recurrent R-spondin fusions in colon cancer. Nature. 2012;488(7413): 660-664.

28. Ferrara R, Auger N, Auclin E, Besse B. Clinical and Translational Implications of RET Rearrangements in Non-Small Cell Lung Cancer. J Thorac Oncol. 2018;13(1): 27-45.

29. Sisdelli L, Cordioli MICV, Vaisman F, Moraes L, Colozza-Gama GA, Alves PAG, et al. AGK-BRAF is associated with distant metastasis and younger age in pediatric papillary thyroid carcinoma. Pediatr Blood Cancer. 2019;66(7): e27707.

30. Ibata M, Iwasaki J, Fujioka Y, Nakagawa K, Darmanin S, Onozawaet M, et al. Leukemogenic kinase FIP1L1-PDGFRA and a small ubiquitin-like modifier E3 ligase, PIAS1, form a positive cross-talk through their enzymatic activities. Cancer Sci. 2017;108(2): 200-207.

31. Stransky N, Cerami E, Schalm S, Kim JL, Lengauer C. The landscape of kinase fusions in cancer. Nat Commun. 2014;5: 4846.

32. Kim SY, Oh SO, Kim K, Lee J, Kang S, Kim K-M, et al. NCOA4-RET fusion in colorectal cancer: Therapeutic challenge using patient-derived tumor cell lines. J Cancer. 2018;9(17): 3032-3037.

33. Jang JS, Lee A, Li J, Liyanage H, Yang Y, Guo L, et al. Common Oncogene Mutations and Novel SND1BRAF Transcript Fusion in Lung Adenocarcinoma from Never Smokers. Sci Rep. 2015;5: 9755.

34. Kim HS, Jung M, Kang HN, Kim H, Park C-W, Kim S-M, et al. Oncogenic BRAF fusions in mucosal melanomas activate the MAPK pathway and are sensitive to MEK/PI3K inhibition or MEK/CDK4/6 inhibition. Oncogene. 2017;36(23): 3334-3345.

35. Catic A, Kurtovic-Kozaric A, Johnson SH, Vasmatzis G, Pins MR, Kogan J. A novel cytogenetic and molecular characterization of renal metanephric adenoma: Identification of partner genes involved in translocation t(9;15)(p24;q24). Cancer Genet. 2017;214-215: 9-15. 
36. Weinberg RA. Cytoplasmic signalling circuitry programs; many of the traits of cancer. In The biology of cancer. $15^{\text {th }}$ edition. Garland Science, Taylor \& Francis group. 2007: 159-207.

37. Maliga Z, Junqueira M, Toyoda Y, Ettinger A, Mora-Bermúdez F, Klemm RW, et al. A genomic toolkit to investigate kinesin and myosin motor function in cells. Nat Cell Biol. 2013;15(3): 325-334.

proto-oncogene

Tissue

atlas.

The

human

protein

atlas. https://www.proteinatlas.org/ENSG00000165731-RET/tissue

39. Gandhi M, Medvedovic M, Stringer JR, Nikiforov YE. Interphase chromosome folding determines spatial proximity of genes participating in carcinogenic RET/PTC rearrangements. Oncogene. 2006;25(16): 2360-2366.

40. Nikiforova MN, Stringer JR, Blough R, Medvedovic M, Fagin JA, Nikiforov YE. Proximity of chromosomal loci that participate in radiation-induced rearrangements in human cells. Science. 2000;290(5489):

41. Wu PH, Gilkes DM, Phillip JM, Narkar A, Cheng TW-T, Marchand J, et al. Single-cell morphology encodes metastatic potential. Sci Adv. 2020;6(4): eaaw6938.

42. Tang NH, Okada N, Fong CS, Arai K, Sato M, Toda T. Targeting Alp7/TACC to the spindle pole body is essential for mitotic spindle assembly in fission yeast. FEBS Lett. 2014;588(17): 2814-2821.

43. Merolla F, Pentimalli F, Pacelli R, Vecchio G, Fusco A, Grieco M, et al. Involvement of H4(D10S170) protein in ATM-dependent response to DNA damage. Oncogene. 2007;26(42): 6167-6175. and is required for proper execution of the DNA damage response in human cells. Cancer Res. 2012;72(8): 2006-2016. 


\section{FGFR3-TACC3}

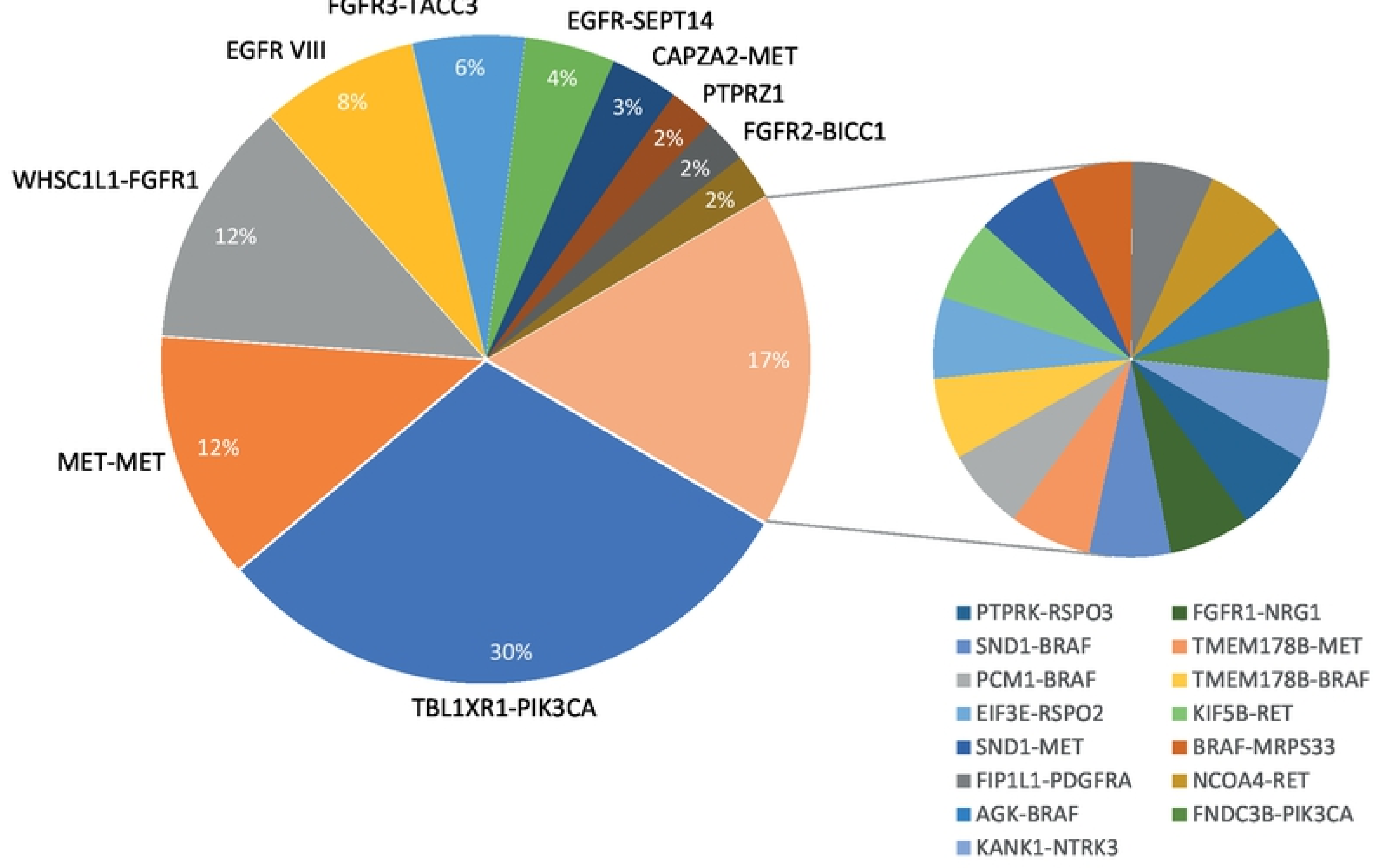

Figure 1. Actionable fusion gene landscape in solid tumours.

Figure 1 

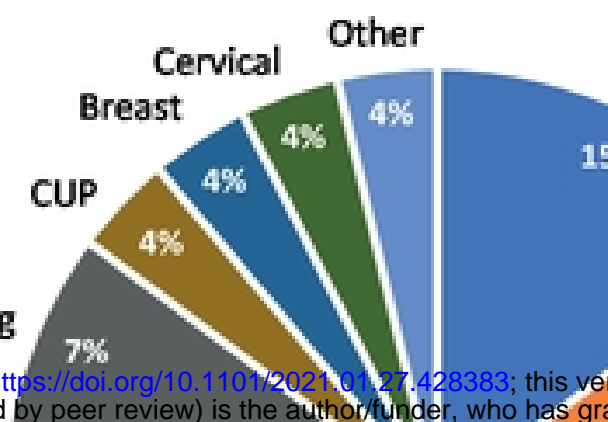

Pancreatic
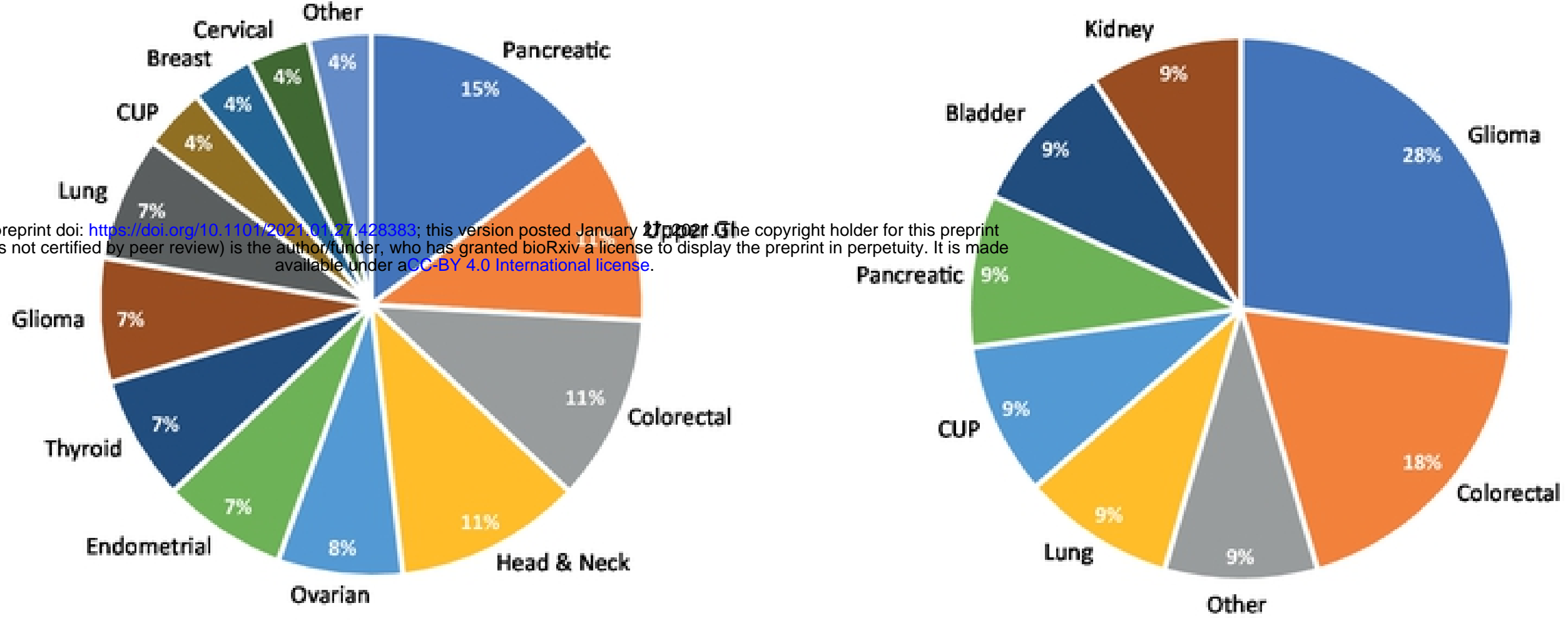

Pancreatic
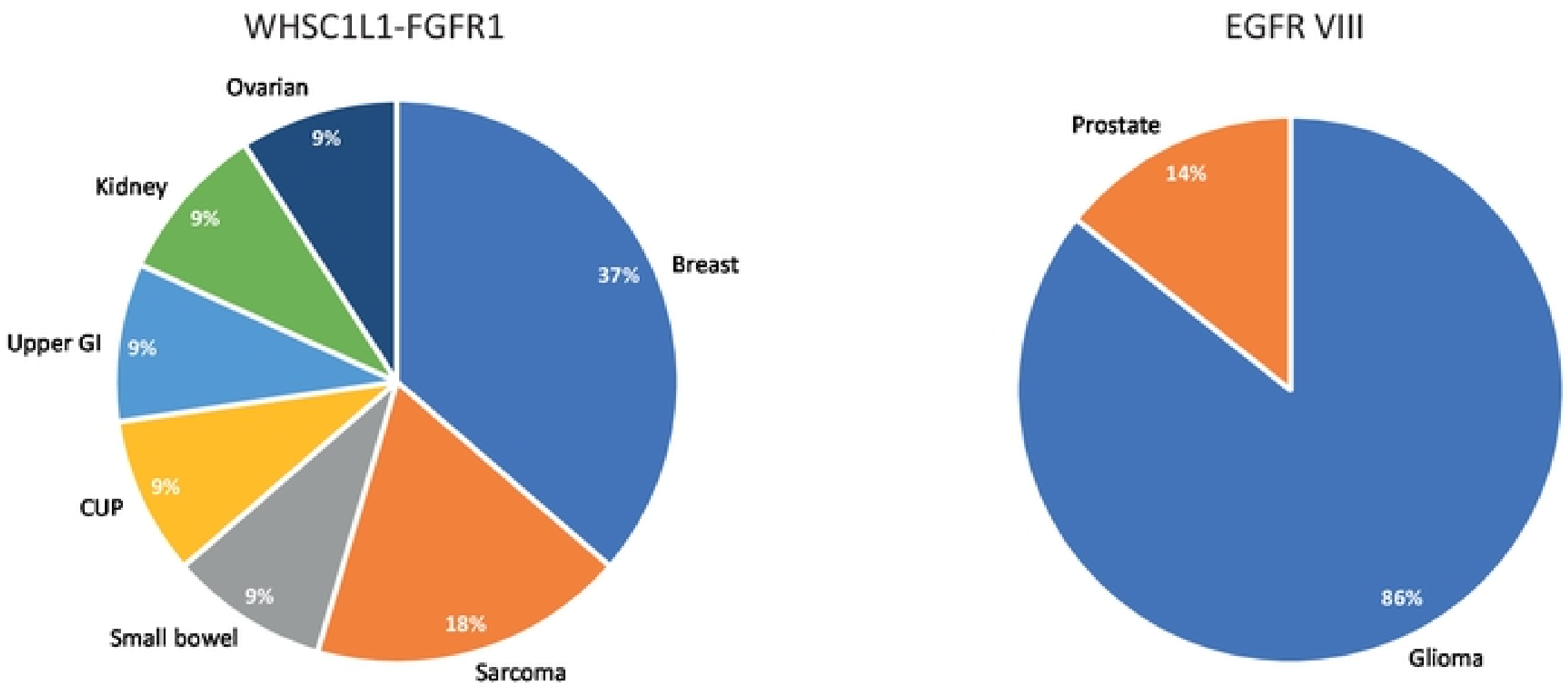

Figure 2. Frequency of the four most common actionable gene fusions in solid tumours. 

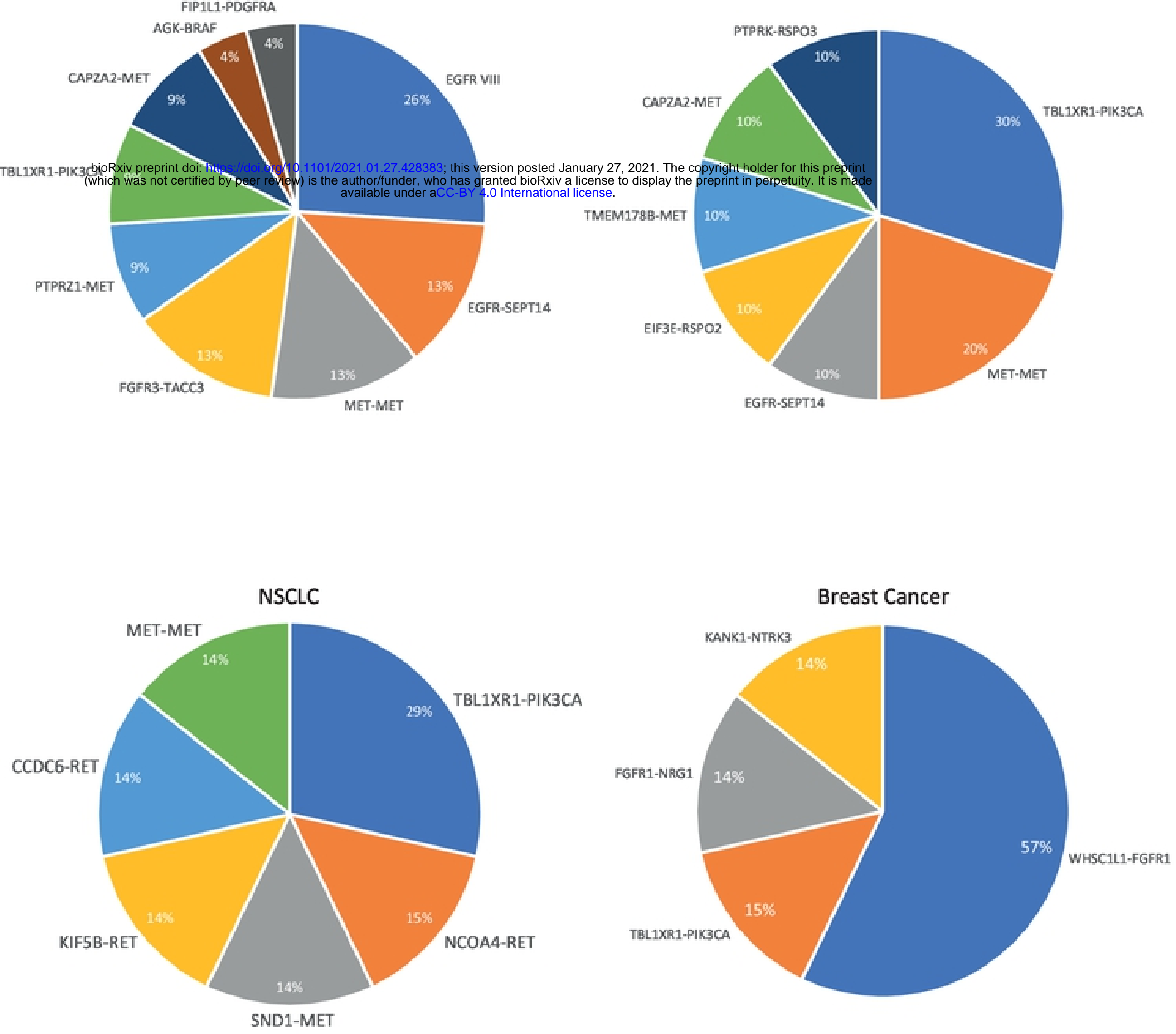

Figure 3. Frequency of actionable gene fusions in glioblastoma, colorectal, NSCLC and breast cancers.

\section{Figure 3}

